\title{
PEERING THROUGH A DIRTY WINDOW: A BAYESIAN APPROACH TO MAKING MINE DETECTION DECISIONS FROM NOISY DATA*
}

$$
\text { CONF-981019-- }
$$

\author{
Stephen W. Kercel \\ Oak Ridge National Laboratory \\ P.O. Box 2008 \\ Oak Ridge, Tennessee 37831-6011 \\ (423) $574-5278$
}

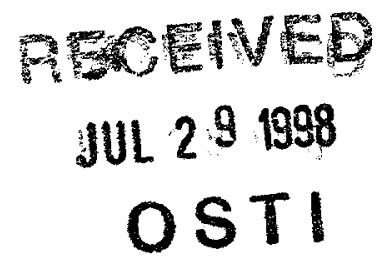

To be presented at the

1998 IEEE International Conference on Systems, Man, and Cybernetics

La Jolla, California

October 11-14, 1998

\begin{abstract}
The submitted manuscript has been authored by a contractor of the U.S.
Govemment under contract No. DE-AC05-960R22464. Accordingly, the

U.S. Covemment retains a nonexclusive, royalty-free license to pubish or

reproctuse the published form of this contribution, or allow others to do so,

for U.S. Govermment purposes."
\end{abstract}

\section{DISTPBUTION OF THS DOOUHENT}
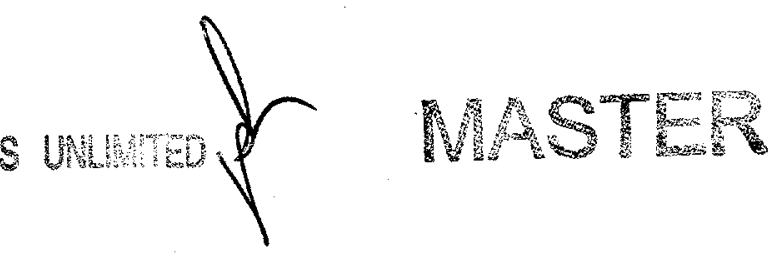

*This research was performed at OAK RIDGE NATIONAL LABORATORY, managed by LOCKHEED MARTIN ENERGY RESEARCH CORP. for the U.S. DEPARTMENT OF ENERGY under contract DE-AC05-960R22464. 


\section{DISCLAIMER}

This report was prepared as an account of work sponsored by an agency of the United States Government. Neither the United States Government nor any agency thereof, nor any of their employees, make any warranty, express or implied, or assumes any legal liability or responsibility for the accuracy, completeness, or usefulness of any information, apparatus, product, or process disclosed, or represents that its use would not infringe privately owned rights. Reference herein to any specific commercial product, process, or service by trade name, trademark, manufacturer, or otherwise does not necessarily constitute or imply its endorsement, recommendation, or favoring by the United States Government or any agency thereof. The views and opinions of authors expressed herein do not necessarily state or reflect those of the United States Government or any agency thereof. 


\section{DISCLAIMER}

Portions of this document may be illegible electronic image products. Images are produced from the best available original document. 


\title{
PEERING THROUGH A DIRTY WINDOW: A BAYESIAN APPROACH TO MAKING MINE DETECTION DECISIONS FROM NOISY DATA
}

\author{
Stephen W. Kercel \\ Instrumentation and Controls Division \\ Oak Ridge National Laboratory \\ PO Box 2008 MS 6011 \\ Oak Ridge Tennessee 37831-6011, USA
}

\begin{abstract}
For several reasons, Bayesian parameter estimation is superior to other methods for extracting features of a weak signal from noise. Since it exploits prior knowledge, the analysis begins from a more advantageous starting point than other methods. Also, since "nuisance parameters" can be dropped out of the Bayesian analysis, the description of the model need not be as complete as is necessary for such methods as matched filtering. In the limit for perfectly random noise and a perfect description of the model, the signal-to-noise ratio improves as the square root of the number of samples in the data. Even with the imperfections of real-world data, Bayesian approaches this ideal limit of performance more closely than other methods.
\end{abstract}

A major unsolved problem in landmine detection is the fusion of data from multiple sensor types. Bayesian data fusion is only beginning to be explored as a solution to the problem. In single sensor processes Bayesian analysis can sense multiple parameters from the data stream of the one sensor. It does so by computing a joint probability density function of a set of parameter values from the sensor output. However, there is no inherent requirement that the information must come from a single sensor.

If multiple sensors are applied to a single process, where several different parameters are implicit in each sensor output data stream, the joint probability density function of all the parameters of interest can be computed in exactly the same manner as the single sensor case. Thus, it is just as practical to base decisions on multiple sensor outputs as it is for single sensors. This should provide a practical way to combine the outputs of dissimilar sensors, such as ground penetrating radar and electromagnetic induction devices, producing a better detection decision than could be provided by either sensor alone.

\section{INTRODUCTION}

Despite the profusion of sensor technologies being applied to the problem, the fact remains that countermine sensor technology has simply not progressed to a satisfactory level. The essence of the present state of countermine sensing is captured in a quote from Colonel Robert Greenwalt, "Today, highly trained, scared soldiers use all their senses, augmented with a coin detector and a stick." [1] Based on the comments of US Army countermine researchers in the literature and informal discussions between Army and Oak Ridge National Laboratory (ORNL) researchers, it appears that the Army is convinced that no single sensing technology is completely satisfactory for mine detection and sees the ultimate solution in the fusion of data from multiple sensor types. An excellent account of the state-of-the-art in multisensor mine detector data fusion is provided by Hanshaw. [2] He considers the present state-of-the-art to be unsatisfactory, that reliable data fusion is unattainable with present software methods, and may not be attainable at all. If successful data fusion is attainable at all, the data from multiple sensors must be spatially correlated within real-world constraints.

Hanshaw defines fusion as the merging of several different data streams of differing importance into a state of awareness of whether or not a mine is present. A good fusion system would mimic animal reaction. Multi-sensor fusion is needed to simultaneously achieve high probability of detection, $P_{d}$, and low probability of false alarm, $P_{f a}$, thus breaking the universal tradeoff between these probabilities that plagues single sensor mine detectors. [3] For combat minefield breaching, both low $P_{d}$ and high $P_{f a}$ are equally deadly. Low $P_{d}$ is the failure to detect mines 
that are actually present. High $P_{f g}$ causes unnecessary halts in moving military formations, increasing their vulnerability to enemy fire.

Some of the specific elements needed to solve the datafusion problem are reasonably well known. The problem in mine detection is "identity data-fusion," and conventional data-fusion methods address it with classic pattern-recognition algorithms. [4] Often these use neural nets with orthogonal data streams, [3] or even more crudely, sometimes they are based on simple thresholding of vector quantities. [5]

Despite Hanshaw's pessimism, there is reason to believe that the mine detector multi-sensor data-fusion problem is tractable. Dogs can consistently detect mines, and this suggests that mines must have detectable features. [6] Whatever form they may take, characterizing features exist and are identifiable. Notwithstanding the argument that a consistent set of characterizing features exists, and notwithstanding the fact that many have tried to find it, nobody has yet found a set of features that reliably characterizes mine identity. This strongly suggests that the features have not been found because researchers have been looking for the wrong thing.

\section{THEORETICAL BACKGROUND}

The argument is commonly, and reasonably, put forward by countermine researchers that if dogs can detect mines, then mines are detectable. Appeal to this argument is the usual justification for the development of electronic systems that seek to emulate the dog's nose. However, there is at least one account of a successful mine detecting dog, who, to all practical effect, does not have a nose. [7] This suggests that what should be emulated by an electronic system is the process by which the dog (or any biological system) fuses multiple sensory cues to identify the presence of a mine.

Does the idea of computational emulation of a biological process actually provide a practical basis for a novel approach to countermine data-fusion? Recent research sponsored by Landauer and Bellman suggests that, in principle, it does. [8] Biological systems process sensory symbols to gain awareness of their environment, and their processing skill improves with experience. They commonly use the data inferred from these symbols to perform classification and grouping, and they do not do so by identifying boundaries between classes. The way that biological systems perform classification suggests that there exists a unifying principle of classification that is applicable to computational systems. [9]

Landauer and Bellman define semiotics as "the study of the appearance (visual or otherwise), meaning, and use of symbols and symbol systems." From their examination of classification by biological systems, they conclude that it would require a radical shift in how symbols are represented in computers to emulate the biological classification process in hardware. However, they argue that semiotic theory should provide the theoretical basis for just such a radical shift. Landauer and Bellman do not claim to have discovered the unifying semiotic principle of patternrecognition, but they suggest that it must be inductive in character. [10]

Indeed, the development of a unified inductive-learning model is the key to artificial intelligence. [11,12] Induction is defined as a mode of reasoning that increases the information content of a given body of data. The application to pattern-recognition in general (and to the mine sensor fusion problem in particular) is obvious. An inductive pattern recognizer would learn the common characterizing attributes of all (possibly infinitely many) members of a class from observation of a finite (preferably small) set of samples from the class, and a finite set of samples not from the class. The problem arises due to the fact that none of the commonly used "learning" paradigms are actually capable of performing induction.

\section{BAYESIAN INDUCTION}

How should this induction be performed? Both the leading thinkers in machine learning and the leading thinkers in smart mine detection believe that it should somehow emulate the process used in biological systems. That process appears to be model-based. Rosen explains the anticipatory behavior of animals in terms of interacting models. [13]

In fact, he defines a formal anticipatory system (a mathematical formulation that exhibits anticipatory behavior) as having five attributes. An anticipatory system, $S_{2}$, must contain the model, $M$, of another system, $S_{1}$. The anticipatory system, $S_{2}$, must contain a set of observables that can be linked mathematically 
to $S_{1}$, and an orthogonal set that cannot. The predictions of the model, $M$, can cause an observable change in $\mathrm{S}_{2}$. There must be some observable difference in the interaction between $S_{1}$, and $S_{2}$ when the model is present, and when it is not. Finally, $M$ must be predictive; based on present knowledge, $M$ must change state faster than $S_{1}$, such that M's changed state constitutes a prediction about $S_{1}$. The point of this discussion is that intelligent behavior is model-based, and in the absence of models, there is no intelligent behavior. More to the point, these models must bear some resemblance to physical reality if the behavior of the intelligent system is to have utility in the real world.

What is the best way to obtain these models? The simple answer is to observe reality to a finite extent, and then to generalize from the observations. To do so is inherently to add information to the data, or to perform an induction. It requires the generation of a likely model based on incomplete information, and the model may be refined in the light of increased knowledge.

How might this be done in practice with noisy data? The most powerful method is Bayesian parameter estimation. [14] Bayesian analysis drops irrelevant parameters, without loss of precision in describing relevant parameters. It fully exploits prior knowledge. Most important, the computation of the most probable values of a parameter set incidentally includes the measure of the probability. That is, the calculation produces an estimate of its own goodness. By comparing the goodness of alternative models, the best available description of the underlying reality is obtained. This is the optimal method of obtaining a model from experimental data, or of predicting the occurrence of future events given knowledge from the past, and of improving the prediction of the future as knowledge of the past improves. [15] Bayesian parameter estimation is a straightforward method of induction.

\section{MINE SENSOR DATA FUSION}

The idea that Bayesian parameter estimation can increase information content by fusing data from multiple sources has been known for some time. $[16,17]$ Inguva, et al. have used parameter estimation to identify underground objects. [18] Collins et al. have used Bayesian methods to refine the operation of single sensor mine detection technologies.[19] However, it has not yet been used for data fusion in mine detection using multiple sensor types.

To get an idea of how Bayesian parameter estimation might work with multiple mine sensors, consider a recent problem for which ORNL has had tangible success. The Enclosed Space Detection System (ESDS), or heartbeat detector, is a device that finds a passenger concealed in a vehicle by identifying a signature caused by the subject's heartbeat.[20] The signature is extracted from the noisy data provided by a geophone placed on the vehicle. The problem is that a moderate wind blowing on the vehicle can create a strong signal that overwhelms the desired signature. The problem is similar to mine detection in that the desired signal is very weak, leading to an unfavorable signal-to-noise ratio, and is obscured by extremely strong deterministic clutter.

The Bayesian approach to the wind problem in ESDS is as follows. The spectrum of the response to wind is very broadband. On the other hand, the vehicle signature resulting from the heartbeat is very narrowband. The heartbeat energy is a series of impulses that excites the natural mechanical resonance of the vehicle, producing a signature whose energy tends to be concentrated in sinusoids at a relatively few discrete frequencies. Bayesian analysis exploits this distinction by using a model consisting of the sum of 50 sinusoids, all falling somewhere between 6 and $16 \mathrm{~Hz}$. Bayesian analysis finds the 50 most probable sinusoidal frequencies (the global maximum of a 50dimensional joint probability density function). Even if the wind is strong, if no heartbeat is present the strongest 50 discrete components of this broadband signal have very low total energy. On the other hand, if the heartbeat is present, the energy will be high, because the 50 discrete sinusoids contain practically all the energy in the signal. The decision is made by computing the energy (sum of squares of amplitudes) in the 50 sinusoids. If the energy exceeds a prescribed threshold, the system indicates that an intruder is hidden in the vehicle.

How does this relate to mine sensor data fusion? In ESDS, the process estimates 50 parameters from the sensor data. The process computes a joint probability density function of a set of parameter values from a single sensor output. However, there is absolutely no requirement that the information must come from a 
single sensor. If multiple sensors are applied to a single process, where several different parameters are implicit in each sensor output data stream, the joint probability density function of all the parameters of interest can be computed in exactly the same manner as the single sensor case. Thus, it is just as practical to base decisions on multiple sensor outputs as it is for single sensors.

To envision how this might enhance the performance of the present generation of mine sensors, imagine the following. Suppose that there are two sensors available, an electromagnetic induction detector (EMI), and a ground penetrating radar (GPR). Suppose that it is known that when a mine is present, the EMI produces a signal that decays exponentially in time, and is characterized by the decay rate. Suppose that it is also known that when a mine is present, the GPR produces a signal characterized as a parabola in space, and can be completely described by three parameters. Also suppose that the two signals are correlated (i.e. when a mine is present, both detectors produce a signature). Finally, suppose that the reliability of both sensors is reduced by noise, and that the objective of the fusion is to obtain a reliable decision when the individual sensors are being corrupted by noise and are saying "maybe." The entire system is characterized by two functions and four parameters. Bayesian analysis of a given data set produced by the system would produce an estimate of the values of the four parameters, and a measure of their joint probability. The decision could be as simple as determining whether or not the joint probability exceeds some threshold.

Suppose, in the above example, that for both sensors the propagation through the ground perturbs the sensor signals. This effect is probably described by a transfer function that is characterized by a conductivity value and a dielectric constant. Since the two sensors operate at significantly different frequencies, the effective values of the soil parameters would be totally different for the two sensors. It would require at least four parameters to describe the effect of the soil on the two-sensor system. Thus, a model describing the output of the two-sensor system, including ground effects, might have two functions and eight parameters. Of these eight parameters, only four are of interest, and the four parameters that relate to soil properties could be "integrated out" as "nuisance parameters." Another approach might be to estimate all eight parameters from the data, applying range limits to the soil parameters, based on what is known a priori about the soil type. Either approach might lead to an instrument that would operate successfully over a range of soil conditions, without the need of knowing specific values of the soil parameters in advance.

Bayesian parameter estimation provides one other advantage that many mine detector operators would find invaluable. The probability measure that is always produced by the analysis can be displayed as a confidence factor. As an example of how this might be useful, suppose that one of the sensors in a mine detection array is a variable-frequency GPR, and that by adjusting the frequency, the operator could adjust the tradeoff between resolution and depth of view. Also suppose that, for a given frequency setting, the detector produces an ambiguous result, such as an alarm that has $50 \%$ probability of being valid. In conventional practice, the operator would simply try the reading again and hope for better luck. However, a far safer technique would be for the operator to try the reading at different frequency settings, while observing how the confidence factor changes as the frequency setting is changed. In many cases the process would converge to either a very high or very low probability measure, providing the operator an unambiguous answer.

\section{CONCLUSIONS}

The countermine community has a difficult problem in trying to identify landmine signatures from the output streams of multiple sensors, and to do so with simultaneous high $P_{d}$ and low $P_{f a}$. Conventional pattern-recognition algorithms, which do not actually learn anything, but rather perform curve fitting in a predefined vector space, have so far proven unequal to the challenge. On the other hand dogs, which also rely on multi-sensory cues, do learn (apparently inductively learning a model) to identify mine signatures with high reliability.

Bayesian parameter estimation also inductively learns a model from a limited data set, and is capable of learning a method of multisensor data fusion. It appears to be very promising as a data-fusion engine for an electronic system to identify mine signatures from multiple types of mine sensors. 


\section{ACKNOWLEDGMENTS}

This research was performed at Oak Ridge National Laboratory, managed by Lockheed Martin Energy Research, Inc., for the U.S. Department of Energy under Contract No. DE-AC05-960R22464.

\section{REFERENCES}

[1] Horowitz, P., et. al., New technological approaches to humanitarian demining, Draft Report JSR-96-115, JASON, The MITRE Corporation, McLean VA, August 1996

[2] Hanshaw, T. and Reidy, D.M., "Operational Standoff Mine Detection: Its Technology and Application," in Detection and Remediation Technologies for Mines and Minelike Targets II, Abinash C. Dubey and Robert L. Barnard, Editors, Proceedings of SPIE, Vol. 3079, pp. 432-442 (1997).

[3] Hanshaw, T., "Multi-sensor fusion for the detection of mines and 'mine like' targets," in Detection Technologies for Mines and Minelike Targets, Abinash C. Dubey, Editor, Proc SPIE 2496, pp. 152-158 (1995).

[4] Hall, D.L., Mathematical Techniques in Multisensor Data Fusion, Artech Hoise, Boston (1992).

[5] Bargel, B., Bers, K., Stein, G. and Traeger, G., "Model-based sensor fusion for minefield detection," in Detection Technologies for Mines and Minelike Targets, Abinash C. Dubey, Editor, Proc SPIE 2496, pp. 509-518 (1995).

[6] Dugan, R., "Detection of Landmines and Unexploded Ordinance by Exploitation of the Chemical Signature," presented at DARPA UXO Detection by the Chemical Signature Conference, Arlington VA, 3 June 1996.

[7] Informal discussion with General Carl Stiner, former Commanding Officer of SOCOM.

[8] Landauer, C. and Bellman, K.L., "Semiotics of Constructed Complex Systems," in Intelligent Systems: A Semiotic Perspective, J. Albus, A. Meystel, and R. Quintero, Organizers, pp. 35-40,
National Institute of Standards and Technology, Gaithersburg MD (1996).

[9] Landauer, C., "Constructing Autonomous Software Systems," Workshop on Biologically Inspired Autonomous Systems: Computation, Cognition, and Action, Durham NC (March 4-5, 1996).

[10] Bellman, K.L., "When Intelligence is in Control," in Intelligent Systems: A Semiotic Perspective, J. Albus, A. Meystel, and R. Quintero, Organizers, pp. 10-11, National Institute of Standards and Technology, Gaithersburg MD (1996).

[11] Goldfarb, L. and Nigam, S., "The Unified Learning Paradigm: A Foundation for AI," in Artificial Intelligence and Neural Networks: Steps Toward Principled Integration, V. Honovar and L. Uhr, Editors, Academic Press, Boston (1994).

[12] Goldfarb, L., "Inductive class representation and its central role in pattern recognition," in Intelligent Systems: A Semiotic Perspective, J. Albus, A. Meystel, and R. Quintero, Organizers, pp. 53-58, National Institute of Standards and Technology, Gaithersburg MD (1996).

[13] Rosen, R., Anticipatory Systems: Philosophical, Mathematical, and Methodological Foundations, Pergammon Press, Oxford, (1985).

[14] Bretthorst, L., Bayesian Spectrum Analysis and Parameter Estimation, Springer-Verlag, Berlin, (1988), pp. 1-5.

[15] Jaynes, E.T., "Generalized Scattering," in Maximum-Entropy and Bayesian Methods in Inverse Problems, C.R Smith and W.T. Grandy, Editors, D. Reidel Publishing Co., Holland (1985), pp. 377-398.

[16] Smith, C.R., “A Bayesian Approach to Multisensor Data Fusion," Proceedings of SPIE, Vol. 1699, pp. 285-299 (1992).

[17] Richardson, J.M. and Marsh, K.A., "Fusion of Multisensor Data," The International Journal of Robotics Research, Vol. 7, No. 6, December 1988, pp. 78-96. 
[18] Lesser, D., Johnson, J.M., Sandness, G., Inguva, R., Noffsinger, K., "A Stick Model for Computing Magnetic Anomalies Produced by Manmade Structures," Journal of Environmental and Engineering Geophysics, Vol. 2, p. 89, 1997.

[19] Collins, L., Ping, G., and Carin, L, "An Improved Bayesian Decision Theoretic Approach for Land Mine Detection," IEEE Transactions on

Geoscience and Remote Sensing, in press.

[20] Kercel, S.W., Baylor, V.M., and Labaj, L.E., "Comparison of Enclosed Space Detection System with Conventional Methods," presented at the American Defense Preparedness Association 13 ${ }^{\text {th }}$ Annual Security Technology Symposium, Virginia Beach VA, June 9-12, 1997. 